OPEN ACCESS

Edited by:

Matteo Cianchetti,

Sant'Anna School of Advanced

Studies, Italy

Reviewed by:

Gursel ALICI,

University of Wollongong, Australia

Ali Sadeghi,

Fondazione Istituto Italiano di

Technologia, Italy

*Correspondence:

Rebecca Kramer

rebecca.kramer@yale.edu

Specialty section:

This article was submitted to

Soft Robotics,

a section of the journal

Frontiers in Robotics and Al

Received: 27 June 2017 Accepted: 04 September 2017 Published: 26 September 2017

Citation:

Zhang J, Jackson A, Mentzer $N$ and Kramer R (2017) A Modular, Reconfigurable Mold for a Soft Robotic Gripper Design Activity.

Front. Robot. Al 4:46. doi: 10.3389/frobt.2017.00046

\section{A Modular, Reconfigurable Mold for a Soft Robotic Gripper Design Activity}

\author{
Jiawei Zhang', Andrew Jackson ${ }^{2,3}$, Nathan Mentzer ${ }^{2,3}$ and Rebecca Kramer ${ }^{1,4 *}$ \\ 'School of Mechanical Engineering, Purdue University, West Lafayette, IN, United States, ${ }^{2}$ Polytechnic Institute, Purdue \\ University, West Lafayette, IN, United States, ${ }^{3}$ College of Education, Purdue University, West Lafayette, IN, United States, \\ ${ }^{4}$ Department of Mechanical Engineering and Materials Science, Yale University, New Haven, CT, United States
}

Soft robotics is an emerging field with strong potential to serve as an educational tool due to its advantages such as low costs and shallow learning curves. In this paper, we introduce a modular and reconfigurable mold for flexible design of pneumatic soft robotic grippers. By using simple assembly kits, students at all levels are able to design and construct soft robotic grippers that vary in function and performance. The process of constructing the modular mold enables students to understand how design choices impact system performance. Our unique modular mold allows students to select the number and length of fingers in a gripper, as well as to adjust the internal geometry of the pneumatic actuator cavity, which dictates how and where bending of a finger occurs. In addition, the mold may be deconstructed and reconfigured, which allows for fast iterative design and lowers material costs (since a new mold does not need to be made to implement a design change). We further demonstrate the feasibility of the modular mold by implementing it in a soft robot design activity in classrooms and showing a sufficiently high rate of student success in designing and constructing a functional soft robotic gripper.

Keywords: soft robotics, gripper, design, engineering education, modular

\section{INTRODUCTION}

Robotics has been demonstrated as an effective vehicle for hands-on learning of technical and tinkering skills (Hamner et al., 2008; Desai et al., 2009; Adamchuk et al., 2012). As such, robotics is being rapidly integrated into K-12 education and extracurricular activities, where the education objectives include lessons in mechanics, electronics, programming, problem-solving, and design thinking (McGrath et al., 2008). While there are a host of educational robotics tools, such as Arduino, LEGO Mindstorms, Fischertechniks, and crowd-funded robotics startups emerging on Kickstarter, perhaps the most widespread implementation of educational robotics is through U.S. FIRST (For Inspiration and Recognition of Science and Technology), which includes a competition for high-school students to challenge their ability to design and build a full-scale functional robot. However, these existing approaches do little to expose the next generation to emerging fields of robotics, such as soft robotics.

Our goal in this work is to enable a soft robot design activity that retains the learning outcomes of more traditional robot design activities, but reduces the time, cost, and complexity of implementation. Traditional robotics requires elements such as motors, gears, wires, and computer codes. By contrast, soft robots are constructed from elastomeric polymers, actuated by material deformation and tend to have a more "organic" feel and appearance that can remind students of animals and objects seen in nature. These elements are more closely related to the disciplines of materials science, 
biology, and chemistry instead of traditional engineering disciplines. Soft robots are also mechanically robust and inherently safe for humans to interact with (Laschi and Cianchetti, 2014; Abidi and Cianchetti, 2017), as well as more affordable when compared to most traditional robots (Polygerinos et al., 2015; Della Santina et al., 2017). By shifting the fundamental building blocks and leveraging the advantages of soft robotics, we believe there may be an opportunity to broaden interest in robotics and STEM disciplines among K-12 students.

In this work, we focus on using a modular, reconfigurable mold as the foundation of a soft robot design activity in an entry-level STEM course. While pneumatic soft robotics is an active and complex field of research, the basic concept of filling a balloon-like structure with air to make it move is quite simple. As such, at least one soft robotics activity has been developed and tested with a variety of age groups, ranging from elementaryschool students to high-school students (Finio et al., 2013). The activity adopts the same fabrication process as we do, but it does not allow students to make any modifications to the mold, and focuses on building rather than design. Our approach of using modular, reconfigurable molds uses the same basic materials and concept, while enabling students to change the configuration of the resulting robot.

The concept of modular soft robotics has been demonstrated previously, but only in a laboratory context. Specific examples include modular soft robotic components attached through magnetic connections (Kwok et al., 2014) or mechanical connectors (Onal and Rus, 2012; Ranzani et al., 2013). Although these methods provide a reasonable amount of design freedom during assembly, they involve pre-fabricated soft pneumatic modules and may not provide students with the opportunity to construct soft robots from scratch. Rather than focusing on modular components, in this work, we build upon the existing activity by introducing a modular mold that allows students to participate in the design of non-modular soft grippers. Figure 1 demonstrates the reconfigurability of the mold and also shows an example gripper fabricated with the modular mold gripping a variety of objects. In the rest of this paper, we describe the development of the modular mold, the classroom context for implementation, and the testing criteria and results.

\section{MOLD DESIGN}

Throughout the development of the modular mold, we sought to optimize two metrics: design freedom and success rate. Design freedom allows students to investigate variables that govern the behavior of a system, create a design to optimize performance, and then build, test, and improve the design (International Technology Education Association and Technology for All Americans Project, 2007). By providing multiple degrees of design freedom, we encourage students to explore and be creative, which enables learning outcomes in both design and fabrication-students learn not only how to make soft robots, but also how to design soft robots.

In addition, success rate during implementation is critical for student self-confidence and continued interest in engineering learning (Bandura, 1977, 2006; Britner and Pajares, 2006; Mamaril et al., 2016). Through our preliminary work, we have observed that even a robot that might be considered a "failure" by laboratory standards typically resulted in an organic, "squirmy" motion that students naturally find intriguing. While obtaining a sufficiently high success rate is a focus of the modular mold evaluation, the soft robot design experience also provides a context to emphasize building and iteration. Considering the context of robotics education, our goal is not to eliminate these failure modes, but rather to help students identify the cause and effect of failures, thus enabling the iterative design experience needed and guide students to turn ideas into principles of practice (Kolodner et al., 1998).

While both design freedom and success rate are important, previous mold designs excel in one or the other, but not both (Section A in Supplementary Material). Therefore, our goal in

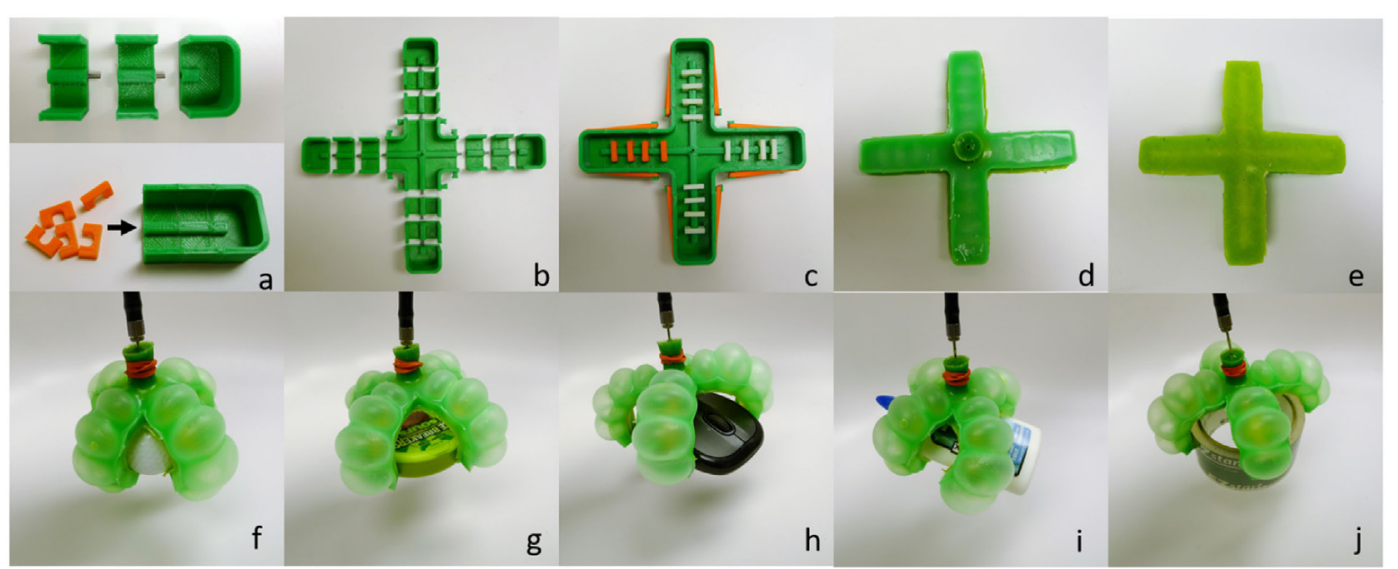

FIGURE 1 | The modular mold kit and a sample soft gripper made from this mold. (A,B) The mold pieces are reconfigurable and many variations of the mold can be built using the same parts; (C) completely assembled mold; (D) top view of the gripper; (E) bottom view of the gripper; (F) picking up a golf ball; (G) picking up a box of candy; (H) picking up a computer mouse; (I) picking up a glue bottle; (J) picking up a roll of tape. 
this work was to increase both metrics in parallel by developing a modular mold. The resulting modular mold comprises six types of mold parts, as shown in Figure 2, but the combination of these parts can result in numerous actuation patterns and behaviors, giving students the opportunity to learn and practice engineering design through iterations. The modular mold kit allows students to select the number of fingers for their gripper design, the number and placement of pneumatic cavities in each finger, and the thickness of the gripper.

The choice of the number of gripper fingers allows students to familiarize themselves with how to make soft robots by experimenting with a single finger before taking on the more difficult task of making an entire soft gripper. Making single soft pneumatic fingers of various designs also provides feedback regarding the success of the designs, which students can then employ to design a full multi-fingered gripper. Furthermore, even in the design of the full gripper, students may vary the number of fingers, thus customizing the gripper to effectively manipulate target objects of specific shapes, sizes, and weights.

An additional highlight of our mold design is the reconfigurable pneumatic networks (often referred to as pneunets in the literature (Ilievski et al., 2011)), which allow curvature of the pneumatic fingers to be designed, as shown in Figure 3. While previous molds have featured static pneumatic networks, we have introduced clips and ridges (Figure 2B) where the clips can be press-fitted to any location on a ridge and may be easily removed and reconfigured. Both the clips and ridges combine to form air chambers in the resulting finger/gripper that define inflation and actuation of the system.

Finally, students may change the thickness of the finger/ gripper by varying the amount of polymer poured into the mold, which will cause more or less bending given a specific pressure. Taken as a whole and compared with previous mold designs, our modular mold provides much more design freedom and flexibility, as summarized in Table $\mathbf{1 .}$

\section{IMPLEMENTATION}

To test the performance of the modular mold, we implemented a soft robotics design activity through a design curriculum in four high-school classrooms, similar to some outreach activities that we had run in the past, as seen in Figure 4. The curriculum was contextualized in a course for 9th-grade students
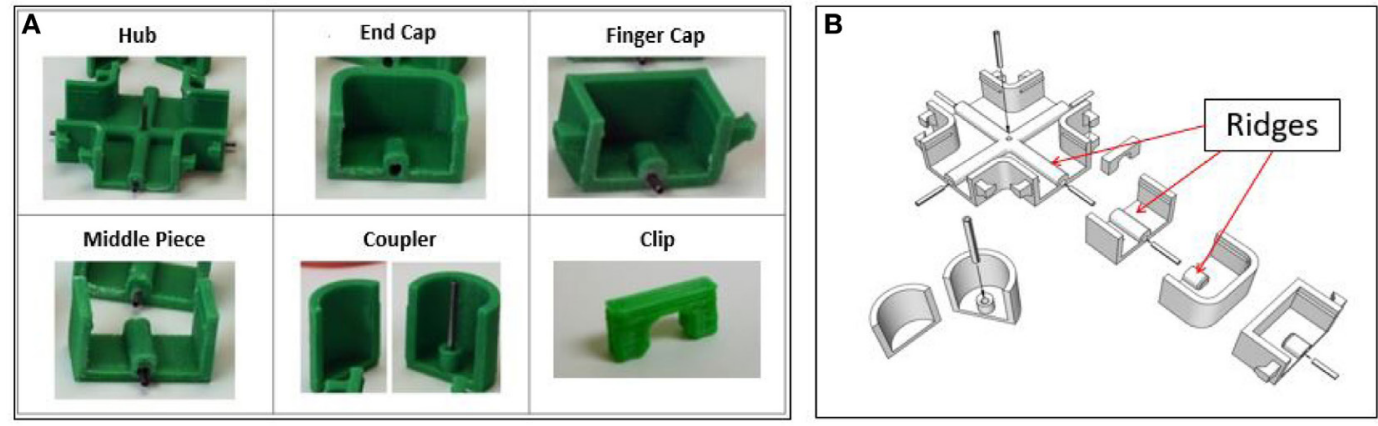

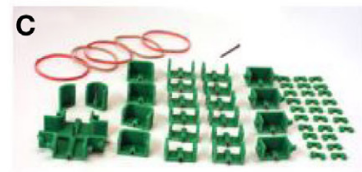

i

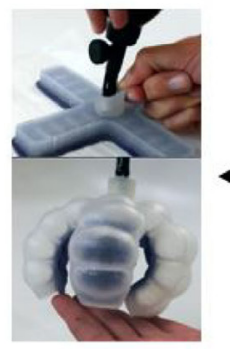

viii

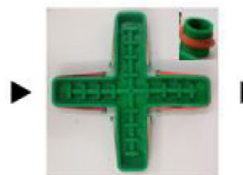

ii

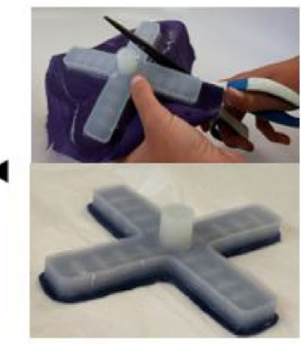

vii

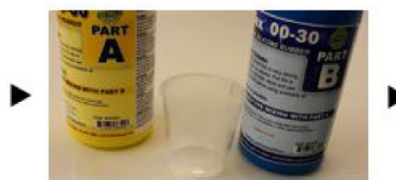

iii

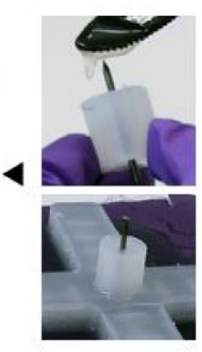

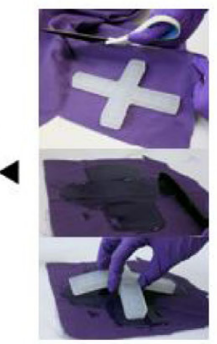

vi

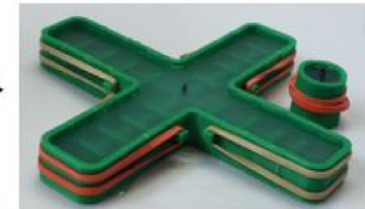

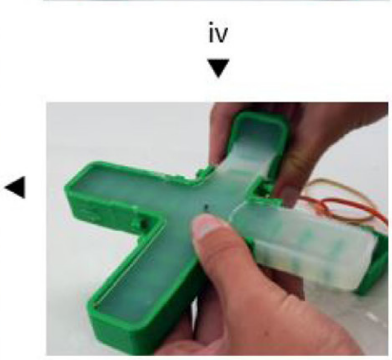

V

FIGURE 2 | Components of the modular mold and its application in the soft robotic gripper design activity. (A) The mold has six different parts, including a hub, end caps, finger caps, middle pieces, a coupler, and clips; (B) an exploded view that shows how the mold is assembled; (C) the eight-step fabrication process of a soft robotic gripper using the modular mold kit; (i) prepare materials; (ii) assemble mold; (iii) prepare silicone elastomer mixture; (iv) pour silicone elastomer into mold and let it cure; (v) remove cured rubber from mold; (vi) apply fabric and attach coupler, let them cure; (vii) trim off extra fabric; (viii) inflate. 

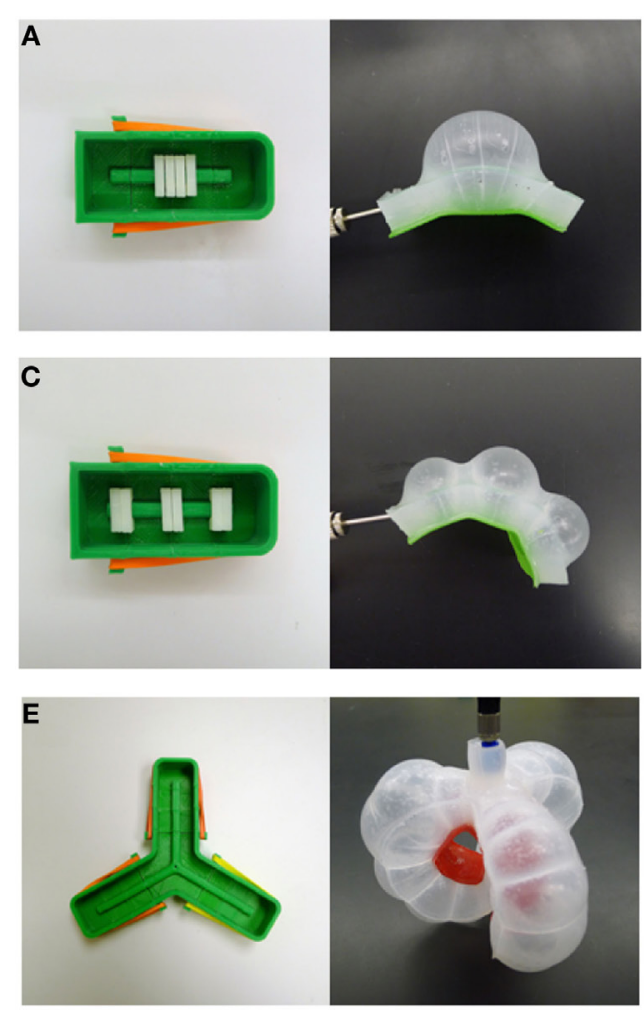
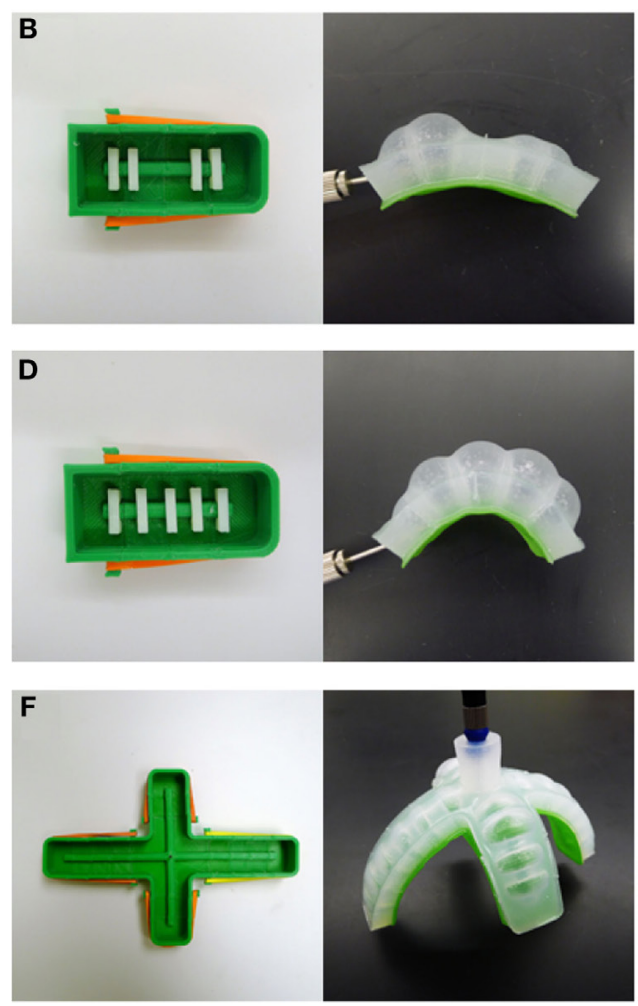

FIGURE 3 | Demonstration of the modular mold's design freedom. On the left are the sample mold configurations, and on the right are the respective finished soft fingers and grippers. (A) A single large pneumatic chamber created by placing four clips together; (B) two sets of clips that are slightly spaced apart; (C) three sets of "double clips" (two clips set next to each other); (D) five evenly spaced clips; (E) three-fingered gripper with equal finger length; (F) four-fingered gripper with different finger length.

TABLE 1 | Degrees of design freedom offered by the modular mold kit.

\begin{tabular}{llc}
\hline Design freedom & Choices & Examples (Figure 3) \\
\hline Robot type & Finger, gripper & A-D vs E,F \\
Number of fingers & 3 or 4 & E vs F \\
Finger length & $0-3$ mid pieces & F \\
Clips & Quantity, spacing & A-D \\
Top layer thickness & Variable & A-F \\
\hline
\end{tabular}

called Foundations of Technology, which is delivered nationally by the STEM Center for Teaching and Learning, a branch of the International Technology and Engineering Educators Association (ITEEA). We note that the work presented herein is part of a larger study aimed at measuring differences in engineering motivation and self-efficacy of students after engaging in either a soft robot design experience or a traditional robot design experience. Development of the modular mold and the measurement of corresponding success rate are foundational to this larger study, as initial success is critical for self-confidence and continued interest in engineering learning (Bandura, 1977, 2006; Britner and Pajares, 2006; Mamaril et al., 2016).

The implementation took about $8 \mathrm{~h}$ to complete over multiple 90-min class sessions. During this time, students designed and fabricated two fingers that varied in design in order to test the

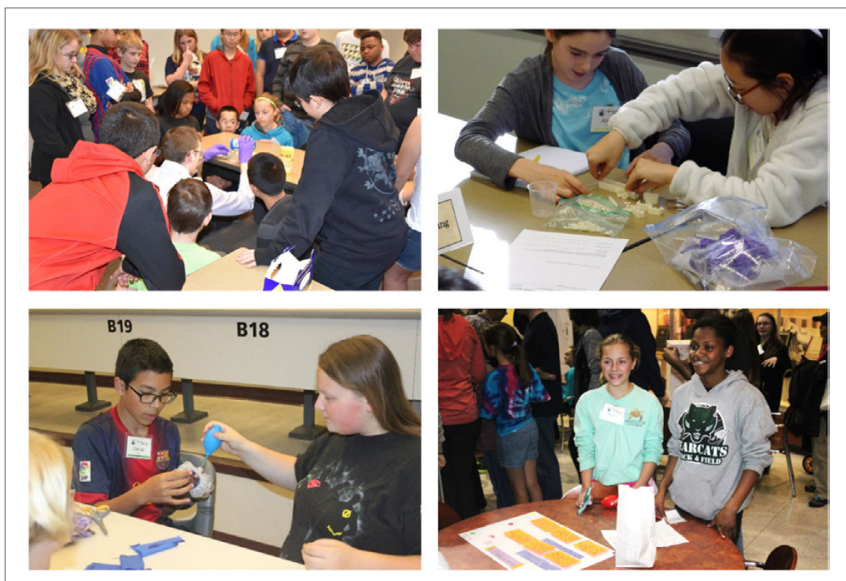

FIGURE 4 | Students participating in the soft robotic gripper design activity at Purdue's Innovation to Reality (I2R) event. Written and informed consent has been obtained from the parents/legal guardians of all depicted individuals for the publication of their children's identifiable images.

impact of variables on finger curvature and performance, then leveraged their findings to design and fabricate a full multifingered soft robotic gripper for the specified task of grasping 
and holding a golf ball for at least $5 \mathrm{~s}$. The majority of students completed the implementation in groups of two, although there were a few groups of three and some students who worked independently, with about $10-15$ groups in each classroom. The implementations were administered by the course teachers, who had attended a professional development meeting prior to the implementations to receive training for the soft robot design activity. Example activity instructions, as well as curriculum materials including a soft robotic gripper design brief and process overview, are provided in the Sections B-D in Supplementary Material.

\section{RESULTS}

Success rate for the activity was evaluated via direct inspection and testing of the student-fabricated grippers by our research team, as well as interviews with participating students and teachers. Success was defined by completion of the design task: if a gripper could grasp and hold a golf ball for $5 \mathrm{~s}$, it was deemed successful. We inspected and evaluated a total of 54 grippers resulting from the classroom implementations. Overall, 29 out of the 54 grippers were successful (a success rate of 54\%), while 25 were unsuccessful. Among the successful grippers, we observed that students utilized the design freedom of the modular mold by adjusting the lengths of fingers, the number of clips inserted, and the spacing between the clips (Figures 5A-D). In addition, multiple teams demonstrated successful grippers by picking up the golf ball and holding it for $5 \mathrm{~s}$ (Figures $5 \mathrm{E}-\mathbf{H}$ ). Thus, the modular mold produced positive results in both design freedom and success rate.

However, we also observed some failed grippers, as shown in Figure 6. The two most common failure modes were clogging and air leaking due to failed seals. Clogging was usually caused by applying an excessive amount of silicone elastomer on the fabric, which would clog the pneumatic actuator cavities, or air channels. Air leaks were usually caused by not applying enough liquid silicone elastomer around the cured silicone elastomer or on the fabric, leaving holes that prevent the grippers from inflating. Another observed failure mode was elastomer rupture, which could be caused by either a non-level curing surface or insufficient filling of the mold. In addition, air bubbles were occasionally identified on the top surface of the gripper, which also contributes to elastomer rupture. Possible causes include mixing the silicone elastomer too vigorously and/or not allowing the silicone elastomer to degas before curing it in the oven. Finally, some cases of failure were a result of overheated molds: as the molds are made of 3D printed thermoplastic, heating the mold near or above the glass transition temperature caused significant mold warping.

We note that all of the above failure modes, accounting for $81 \%$ of all observed failures, are process-related and would have happened for a non-modular mold as well. Only a single failure mode was observed that was related to modularizing the mold. This mold-related failure mode was a result of clips insufficiently connecting to the mold ridges, which in turn caused the clips to detach from the ridges when filling the mold with elastomer. Based on the failure mode data we collected (Table 2), we infer that the modular design had a minimum effect on the success rate. Furthermore, since our gripper inspection was performed after student testing and presentations, some grippers had already failed when we handled them. It is possible that they worked at one point but ruptured later when students overinflated or performed destructive testing on their grippers. Therefore, the success rate presented here is conservative.
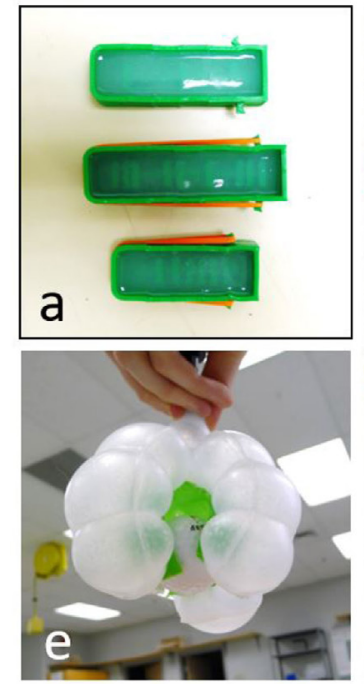
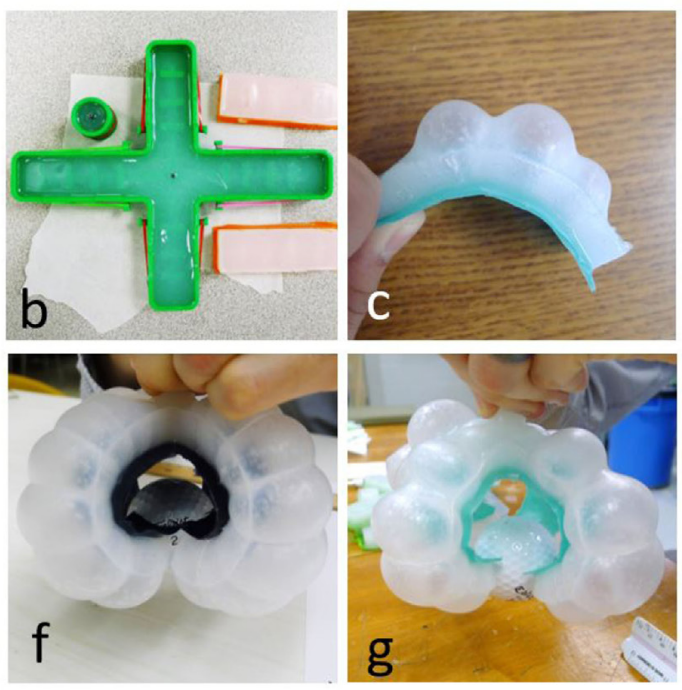

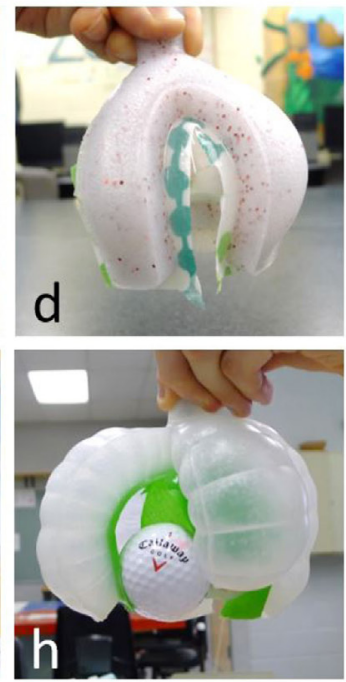

FIGURE 5 | Successful fingers and grippers designed and fabricated by students. (A) Multiple assembled finger molds with different length and different number and spacing of the clips; (B) an assembled gripper mold with different finger lengths and different numbers of clips; (C) a successful soft finger with three air chambers; (D) a successful gripper fabricated by the students without any clips; (E-H) various grippers demonstrating the ability to pick up the golf ball and hold it for $5 \mathrm{~s}$. 

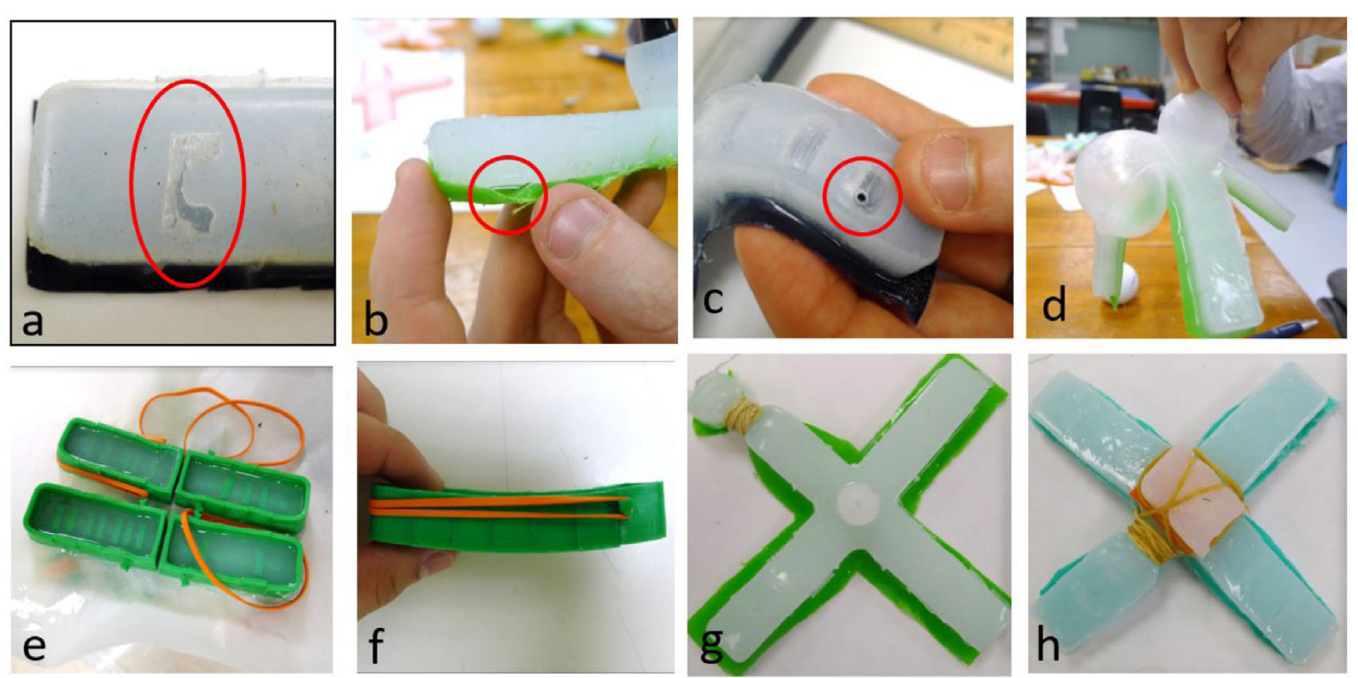

FIGURE 6 | Common failure modes from the classroom implementations and some of students' attempts to repair the grippers. (A) Air leaking from a hole caused by floating clips; (B) air leaking from the side; (C) air leaking from an air bubble; (D) center of the robot was inflated due to multiple clogged air channels; (E) a few warped molds; (F) the warped molds bending upward; (G) students tried to fix an air leaking by tying a rubber band around it; (H) students attached a finger to the gripper instead of using the coupler.

TABLE 2 | Failure mode statistics of the soft robotic grippers.

\begin{tabular}{lcrl}
\hline Failure mode & Quantity & Percent & Cause \\
\hline Air leaking due to failed seals & 15 & 55 & Process \\
Clogging & 7 & 26 & Process \\
Thin top layer due to unlevel curing surface or & 3 & 11 & Process \\
mold warping & & & \\
Air leaking due to floating clips & 1 & 4 & Mold \\
Air leaking due to air bubbles on top layer & 1 & 4 & Process \\
Total & 27 & 100 & \\
\hline
\end{tabular}

Note that two of the 25 failed grippers demonstrated dual failure modes. Therefore, the total number of failures is 27 instead of 25 .

\section{DISCUSSION}

The intent of this research was to produce and test a modular mold for soft robotic fabrication that increased design freedom while maintaining a sufficiently high success rate. On the one hand, classroom observation and gripper inspection indicated that students were indeed using the modularity and design features of the mold pieces to change their designs. For example, students changed the number and spacing of clips and also adjusted the length of the fingers to pick up the specified objects. During the fabrication process, filled lines in the mold helped students control the amount of silicone elastomer and ensured that their mold was curing on a level surface. The reusability of the modular finger and gripper mold pieces allowed students to apply their learning from design and fabrication of fingers to the design and fabrication of full multi-fingered grippers. Finally, the modular mold was quick to assemble and, as a result, students were able to focus on design iterations. Compared to the one-piece mold (Finio et al., 2013), the modular mold has evidently expanded design freedom.
On the other hand, we question whether the success rate achieved was sufficiently high in these initial implementation attempts with the modular mold. Students' achieved success plays an important role in affecting student attitudes and is critical to reaching our broader goal of enhancing student self-efficacy, motivation, and subsequent STEM interest through soft robot design experiences. Although the success rate varied from class to class, the four classes that implemented the soft robot design curriculum achieved an overall success rate of 54\%. Currently, there is no documented success rate for the previous soft robot gripper activity and, as a result, we are not in a position to draw comparisons. Instead, in order to make conclusions, we situate this success rate in relation to motivational theory (Vygotsky, 1987) and measured student outcomes (self-efficacy) that we have previously reported (Jackson et al., 2017).

Vygotsky (1987) introduced the concept of The Zone of Proximal Development (ZPD), which we describe to situate our findings. This theory describes that when a student is within the ZPD for a particular task, providing the appropriate assistance (scaffolding) will enhance the student's abilities enough to successfully complete the task. An appropriately situated instructional task will be challenging enough that it stretches the students, yet includes the necessary resources for them to accomplish the task. In our case, the teachers' instructions, handouts, and process demonstrations (Sections B-D in Supplementary Material) seem to provide this support. Since we have not received any feedback that the activity was too challenging or too straightforward, we argue that we are indeed within the ZPD and that completing such an activity can serve to enhance student abilities (Yorke, 2003).

Further evidence of appropriate challenge in the activity is based on measured outcomes of the activity (Jackson et al., 2017). As part of our broader study, pre- and post-surveys were conducted for both the soft robot design activity described herein 
and a traditional robot design activity with identical learning outcomes. Preliminary survey scores were collected and analyzed to see if there was a change in students' levels of self-efficacy before and after the activity, as well as differences between the types of robotics activity. Based on ANOVA results reported in our other work (Jackson et al., 2017), there was a statistically significant difference in the self-efficacy change over time between the types of robotics activity $\left(p=0.03, \eta^{2}=0.025\right)$ : the mean self-efficacy from the traditional robotics activity decreased $\left(\Delta M_{H}=-0.1\right)$, while the mean self-efficacy from the soft robotic gripper design activity increased $\left(\Delta M_{S}=0.2\right)$. While the practical significance is small, the results are still promising for future iterations. The statistically significant change in self-efficacy supports our conclusion that the modular design is meeting the education objectives because the success rate is sufficiently high to foster students' confidence. When approached carefully, student experiences with design failure can provoke meaningful discussion and learning opportunities (Lottero-Perdue and Parry, 2017). We hope that further changes to the curriculum materials that improve the overall success rate will magnify the effects on student self-efficacy which we have observed.

\section{CONCLUSION}

In this work, we have introduced a modular, reconfigurable mold kit for a soft robotic gripper design activity. We also demonstrated the feasibility of a soft robotics program using this modular mold by showing results from classroom implementations. By breaking down the mold design and giving users the freedom to control several key design parameters, we provide users with a unique design learning experience. Our modular mold kit design also produced a sufficiently high success rate that enabled students to achieve the desired learning outcomes. Furthermore, we have identified common failure modes during fabrication of the soft robotic grippers made from the modular mold kit and provided recommendations to assist users in the iterative design process.

During our school visits, we observed that students were engaged throughout the activity and that the overall experience was positive. Participants also indicated that they were likely to recommend this activity to other fellow students because of the unique, organic characteristics of soft robotics, which differentiates this activity significantly from existing robotics programs. Data collection in the form of feedback and assessments from both teachers and students will further evaluate the reliability of our modular mold to improve learning outcomes such as selfefficacy and motivation for STEM disciplines. We are currently preparing for the second round of classroom implementations, and we expect the success rate to increase as we continue to refine

\section{REFERENCES}

Abidi, H., and Cianchetti, M. (2017). On intrinsic safety of soft robots. Front. Robot. AI 4:5. doi:10.3389/frobt.2017.00005

Adamchuk, V., Barker, B., Nugent, G., Grandgenett, N., Patent-Nygren, M., Morgan, K., et al. (2012). "Learning geospatial concepts as part of a non-formal education robotics experience," in Robots in K-12 Education: A New Technology for Learning, Hershey, PA, 284-300. the modular mold and the curriculum. We will also continue to research the efficacy of the experience as we collect more success rate data. This is the first time that soft robotics has been formally introduced into STEM education, and we believe that this work will be instrumental in advancing current and future robotics programs.

\section{ETHICS STATEMENT}

This study was carried out in accordance with the recommendations of the Purdue University institutional review board. Written and informed consent has been obtained from all adult participants and from the parents/legal guardians of all nonadult participants. The study and the protocol were reviewed and approved by the Purdue University institutional review board.

\section{AUTHOR CONTRIBUTIONS}

RK and NM conceived and designed the research. All authors contributed to the acquisition, analysis, and interpretation of data for the work. JZ and RK wrote the manuscript with critical input from AJ and NM. All authors agreed to be accountable for all aspects of the work in ensuring that questions related to the accuracy or integrity of any part of the work are appropriately investigated and resolved.

\section{ACKNOWLEDGMENTS}

We would like to thank R. Adam Bilodeau, a Ph.D. student in the Purdue Faboratory, for his contribution to this work. We would also like to acknowledge the efforts made by Sarah Hacker, an undergraduate student from Purdue University, and Shae Henry, an undergraduate student from Georgetown College. Sarah Hacker generated multiple mold design concepts and contributed to the early stage development of this work, while Shae Henry spent a summer in the Purdue Faboratory continuing to improve the mold design and also creating a demonstration video that helps first-time users to learn how to make soft grippers.

\section{FUNDING}

This work was supported by the National Science Foundation under Grant DRL-1513175.

\section{SUPPLEMENTARY MATERIAL}

The Supplementary Material for this article can be found online at http://journal.frontiersin.org/article/10.3389/frobt.2017.00046/ full\#supplementary-material.

Bandura, A. (1977). Self-efficacy: toward a unifying theory of behavioral change. Psychol. Rev. 84, 191. doi:10.1037/0033-295X.84.2.191

Bandura, A. (2006). "Guide for constructing self-efficacy scales," in Self-Efficacy Beliefs of Adolescents, eds F. Pajares and T. C. Urdan, Vol. 5 (Greenwich, CT: Information Age Publishing), 307-337.

Britner, S. L., and Pajares, F. (2006). Sources of science self-efficacy beliefs of middle school students. J. Res. Sci. Teach. 43, 485-499. doi:10.1002/ tea. 20131 
Della Santina, C., Piazza, C., Gasparri, G. M., Bonilla, M., Catalano, M. G., Grioli, G., et al. (2017). The quest for natural machine motion: an open platform to fast-prototyping articulated soft robots. IEEE Robot. Autom. Mag. 24, 48-56. doi:10.1109/MRA.2016.2636366

Desai, M., Stubbs, K., Steinfeld, A., and Yanco, H. (2009). Creating trustworthy robots: lessons and inspirations from automated systems. in AISB Convention: New Frontiers in Human-Robot Interaction, Edinburgh.

Finio, B., Shepherd, R., and Lipson, H. (2013). "Air-powered soft robots for k-12 classrooms," in Proceedings of the IEEE Integrated STEM Education Conf. (ISEC), Princeton, NJ, 1-6.

Hamner, E., Lauwers, T., Bernstein, D., Nourbakhsh, I. R., and DiSalvo, C. F. (2008). "Robot diaries: broadening participation in the computer science pipeline through social technical exploration," in AAAI Spring Symposium: Using AI to Motivate Greater Participation in Computer Science, Palo Alto, CA, 38-43.

Ilievski, F., Mazzeo, A. D., Shepherd, R. F., Chen, X., and Whitesides, G. M. (2011). Soft robotics for chemists. Angew. Chem. Int. Ed. Engl. 123, 1930-1935. doi:10.1002/ange.201000255

International Technology Education Association and Technology for All Americans Project. (2007). Standards for Technological Literacy: Content for the Study of Technology. 3rd edn. Reston, VA: International Technology Education Association.

Jackson, A., Mentzer, N., Kramer, R., and Zhang, J. (2017). "Enhancing student motivation and efficacy through soft robot design," in Proceedings of the 2017 ASEE Annual Conference \& Exposition, Columbus, $\mathrm{OH}$.

Kolodner, J. L., Crismond, D., Gray, J., Holbrook, J., and Puntambekar, S. (1998). "Learning by design from theory to practice," in Proceedings of the International Conference of the Learning Sciences, Vol. 98, Atlanta, GA, 16-22.

Kwok, S. W., Morin, S. A., Mosadegh, B., So, J.-H., Shepherd, R. F., Martinez, R. V., et al. (2014). Magnetic assembly of soft robots with hard components. Adv. Funct. Mater. 24, 2180-2187. doi:10.1002/adfm.201303047

Laschi, C., and Cianchetti, M. (2014). Soft robotics: new perspectives for robot bodyware and control. Front. Bioeng. Biotechnol. 2:3. doi:10.3389/fbioe.2014.00003

Lottero-Perdue, P. S., and Parry, E. A. (2017). Elementary teachers' reflections on design failures and use of fail words after teaching engineering for two years. J. Pre Coll. Eng. Educ. Res. 7, 1. doi:10.7771/2157-9288.1160
Mamaril, N. A., Usher, E. L., Li, C. R., Economy, D. R., and Kennedy, M. S. (2016) Measuring undergraduate students' engineering self-efficacy: a validation study. J. Eng. Educ. 105, 366-395. doi:10.1002/jee.20121

McGrath, B., Sayres, J., Lowes, S., and Lin, P. (2008). "Underwater lego robotics as the vehicle to engage students in stem: The build it project's first year of classroom implementation," in American Society for Engineering Education Mid-Atlantic, Hoboken, NJ.

Onal, C. D., and Rus, D. (2012). "A modular approach to soft robots," in 2012 4th IEEE RAS \& EMBS International Conference on Biomedical Robotics and xBiomechatronics (BioRob) (IEEE: Rome), 1038-1045.

Polygerinos, P., Galloway, K. C., Savage, E., Herman, M., O’Donnell, K., and Walsh, C. J. (2015). "Soft robotic glove for hand rehabilitation and task specific training," in 2015 IEEE International Conference on Robotics and Automation (ICRA), (IEEE: Seattle, WA), 2913-2919.

Ranzani, T., Cianchetti, M., Gerboni, G., De Falco, I., Petroni, G., and Menciassi, A. (2013). "A modular soft manipulator with variable stiffness," in 3rd Joint Workshop on New Technologies for Computer/Robot Assisted Surgery, Verona, 11-13.

Vygotsky, L. (1987). Mind in Society: The Development of Higher Psychological Processes, Vol. 5291 (Cambridge, MA: Harvard University Press), 157. Zone of proximal development.

Yorke, M. (2003). Formative assessment in higher education: moves towards theory and the enhancement of pedagogic practice. Higher Educ. 45, 477-501. doi:10.1023/A:1023967026413

Conflict of Interest Statement: The authors declare that the research was conducted in the absence of any commercial or financial relationships that could be construed as a potential conflict of interest.

Copyright (C) 2017 Zhang, Jackson, Mentzer and Kramer. This is an open-access article distributed under the terms of the Creative Commons Attribution License (CC BY). The use, distribution or reproduction in other forums is permitted, provided the original author(s) or licensor are credited and that the original publication in this journal is cited, in accordance with accepted academic practice. No use, distribution or reproduction is permitted which does not comply with these terms. 